\title{
Laboratory experiments as support to the built up of Titan's theoretical models and interpretation of Cassini-Huygens data
}

\author{
Marie-Claire Gazeau ${ }^{1}$, Yves Benilan ${ }^{1}$, Et Touhami Es-sebbar ${ }^{1}$, \\ Thomas Ferradaz $^{1}$, Eric Hébrard ${ }^{1}$, Antoine Jolly ${ }^{1}$, François Raulin ${ }^{1}$, \\ Claire Romanzin ${ }^{1}$, Jean-Claude Guillemin ${ }^{2}$, Coralie Berteloite ${ }^{3}$, \\ André Canosa ${ }^{3}$, Sébastien D. Le Picard ${ }^{3}$, and Ian R. Sims ${ }^{3}$
}

${ }^{1}$ LISA (Laboratoire Interuniversitaire des Systèmes atmosphériques), groupe de Physico-chimie Organique Spatiale, UMR CNRS 7583, Universités Paris 12 et Paris 7, Créteil, 94010, France email: gazeau@lisa.univ-paris12.fr

${ }^{2}$ Sciences chimiques de Rennes, UMR 6226 CNRS-Ecole Nationale Supérieure de Chimie de Rennes, Rennes, F-35700, France

${ }^{3}$ Équipe d'Astrochimie Expérimentale, Université de Rennes 1, France

\begin{abstract}
To interpret the concentrations of the products measured in Titan's atmosphere and to better understand the associated chemistry, many theoretical models have been developed so far. Unfortunately, large discrepancies are still found between theoretical and observational data. A critical examination of the chemical scheme included in these models points out some problems regarding the reliability of the description of critical reaction pathways as well as the accuracy of kinetic parameters. Laboratory experiments can be used to reduce these two sources of uncertainty. It can be:

i) experimental simulations: in our laboratory (LISA), representative Titan's simulation experiments are planned to be carried out in a reactor where the initial gas mixture will be exposed, for the first time, to both electrons and photons. Thus, the chemistry between $\mathrm{N}$ atoms and $\mathrm{CH}_{3}, \mathrm{CH}_{2}$, $\mathrm{CH}$ fragments, issued from electron dissociation of $\mathrm{N}_{2}$ and photo-dissociation of $\mathrm{CH}_{4}$ respectively, will be initiated. Thank to a time resolved technique, we will be able to analyse "in situ", qualitatively and quantitatively, the stable species as well as the short life intermediates. Then, the implied chemistry will be determined precisely, and consequently, its description will be refined in theoretical models. The current status of this program will be given.

ii) specific experiments: they are devoted, for example, to determine kinetic rate constants and low temperature VUV spectra that will be used to feed models and to interpret observational data. Such experiments performed in LISA and in Rennes' laboratory concern polyynes and cyanopolyynes as these compounds could link the gaseous and the solid phase in planetary atmosphere. Results concerning $\mathrm{C}_{4} \mathrm{H}^{+}$hydrocarbons kinetic rate constants and VUV cross section of $\mathrm{HC}_{3} \mathrm{~N}$ and $\mathrm{HC}_{5} \mathrm{~N}$ will be detailed.
\end{abstract}

\section{Discussion}

ZIURYS: This is a very nice measurement of hydrocarbon radical chain reactions. The rate measurements are very difficult experiments and they also have interstellar applications. What do you think is more important for the formation of these poly-ions in Titan's atmosphere, radical-radical reactions or ion-molecule reactions?

Gazeau: In fact, it depends on what you are interested in. There is chemistry that is important in the ionosphere, but this is not the same chemistry as that occurring lower in the atmosphere. I am interested in the stratospheric chemistry. 


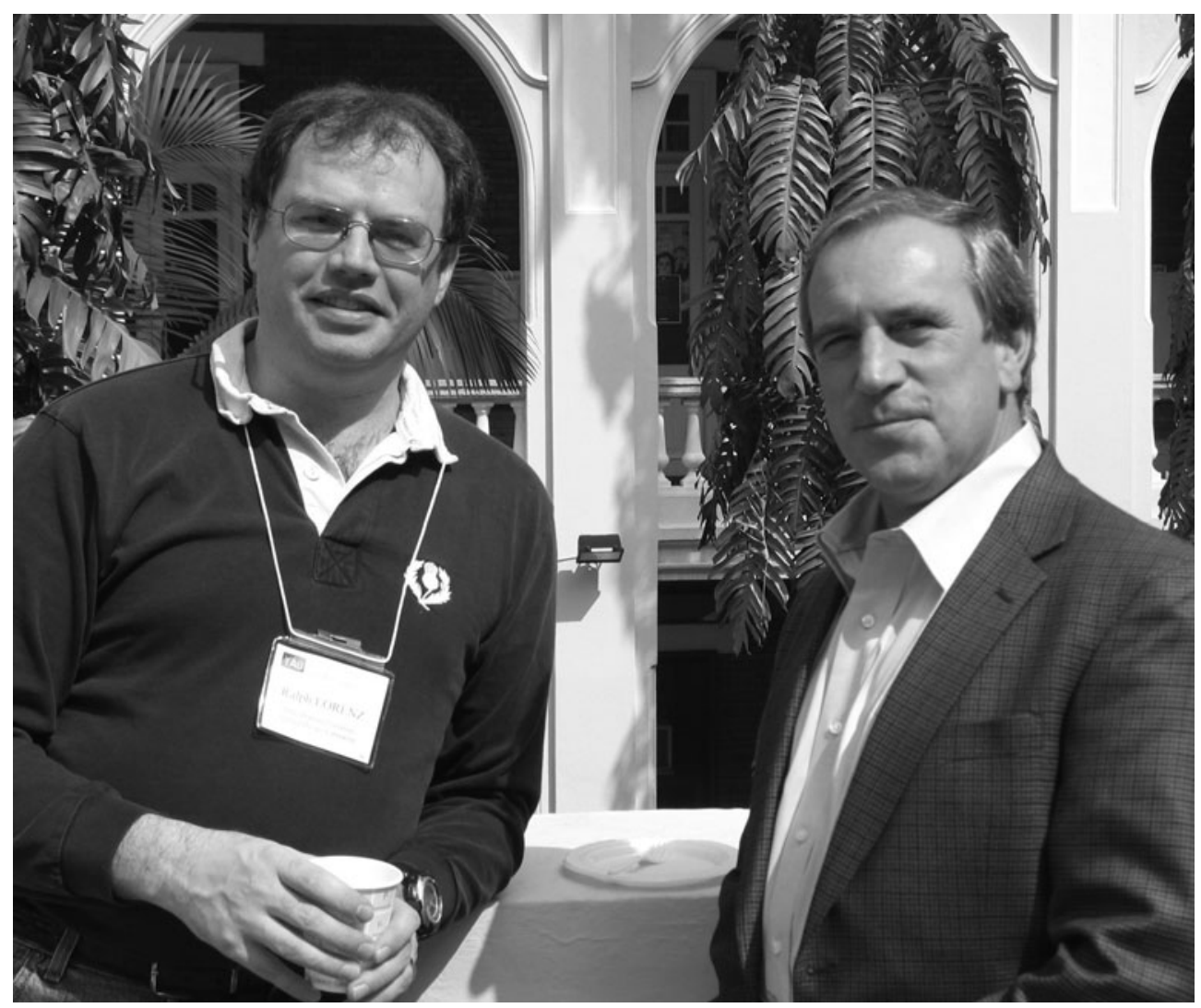

Ralph Lorenz (left) and J. Hunter Waite (right) (photo by Dale Cruikshank). 